\title{
Dually-Eligible Working-Age Adults with Disabilities: Issues and Challenges as Health Reform is Implemented
}

\author{
By Jean P. Hall, PhD \\ Institute for Health and Disability Policy Studies, University of Kansas, Lawrence, KS
}

\begin{abstract}
Acknowledgements
The content of this article is based on information presented at the AcademyHealth Disability Research Interest Group (DRIG) meeting held in Orlando, FL, June 23, 2012, with funding from The Commonwealth Fund. The author would like to thank René Jahiel for his lifetime contributions to the DRIG, Tracy Rausch McDowell for her work in making the meeting happen, and Allen Jensen and Craig Thornton for serving as discussants.
\end{abstract}

This article was originally published in 2013 by Disability and Health Journal, Volume 6, Issue 2, pages 72-74. DOI: 10.1016/j.dhjo.2013.01.004 


\begin{abstract}
This special section on working-age adults with disabilities dually eligible for Medicare and Medicaid is based on two panel presentations at the June 2012 meeting of the AcademyHealth Disability Research Interest Group. The papers included here consider the issues of health care access and outcomes as well as employment for this group of approximately four million individuals, many with complex health care needs and high costs. ${ }^{\mathrm{i}}$
\end{abstract}

\title{
Background
}

The Disability Research Interest Group (DRIG) at AcademyHealth is dedicated to coordination, quality improvement, and financing of services, assistive devices, and environmental changes needed for the optimal health and social integration of people in situations of disability. The DRIG meets annually in conjunction with the AcademyHealth Annual Research Meeting, typically hosting one or more panel presentations related to disability and health. In 2011, the DRIG received funding through the Dual Eligibles Initiative of The Commonwealth Fund to convene webinars and the annual meeting, specifically focusing on issues related to the experiences of the under-65 population with disabilities dually eligible for Medicare and Medicaid. The Dual Eligibles Initiative seeks to bring about better care, improved care experiences, and reduced costs for these beneficiaries through better coordination of services.

Research related to this younger dually-eligible population is particularly important as federal and state policymakers seek to better coordinate care and achieve cost savings across the two programs. Despite the fact that younger individuals with disabilities comprise approximately $43 \%$ of the total population dually eligible for Medicare and Medicaid, most health services and policy research on duals focuses only on those ages 65 and older, or considers all duals as a 
single group. ${ }^{1}$ Yet, younger duals are different from seniors in many important and policyrelevant ways, including the types of medical conditions they experience, the types of Medicaid and Medicare services they utilize and the relative costs for these services, their potential to remain on public assistance and benefits for decades, and their ability to engage in long-term competitive employment given the right incentives and supports.

\section{The Papers}

The 2012 DRIG meeting was held on June 23 in Orlando. The DRIG invited abstracts for presentations, with an emphasis on younger duals. The eight papers accepted for panel presentations fell naturally into two overarching categories: Employment and Health Outcomes. Papers based on five of these presentations are included in this issue. Even though papers were submitted independently, several common themes emerged across both the Employment and Outcomes panels. As might be expected, the Affordable Care Act (ACA) was mentioned in many of the presentations as a critical policy measure with tremendous potential to affect the health and employment of the dually-eligible population. Several speakers also noted the importance and possible benefits of better coordinated care. And, virtually all speakers touched on the important and essential role of employment in controlling costs and improving outcomes for individuals who, unlike most seniors, may otherwise be on public assistance and health insurance for many decades if not gainfully employed.

\section{Employment Panel}

Dr. Jerry Riley of the Center for Medicare and Medicaid Innovation at the Centers for Medicare and Medicaid Services (CMS) opened the Employment Panel by discussing his investigation of cash benefit and health care expenditure patterns for disabled adults under age 65 using linked federal datasets. ${ }^{\text {ii }}$ This presentation reminded the audience that while health care 
expenditures account for a large portion of federal costs for adults with disabilities, cash supports through SSDI and SSI also play a major role. To the extent that people with disabilities apply for Social Security cash benefits (SSI/SSDI) as a gateway to health insurance, greater availability of affordable health insurance under health reform may reduce disability applications and costs for both Social Security and Medicare/Medicaid. The presentation also noted that duals had the highest overall medical costs and highlighted various federal initiatives under health reform to encourage better coordinated care for this population. Finally, the authors suggested that an analysis of overall expenditures, including cash benefits and health care costs, would yield a more accurate picture of the potential savings from employment incentives and supports, including improved health outcomes.

Next, presenter Dr. Alice Levy from George Washington University used a simulation model to predict the impact of the ACA on working-age adults with disabilities. She and her coauthors estimated that more than 2 million adults with disabilities will gain coverage through the various ACA coverage expansions and that, unlike currently, coverage rates will be higher among the employed. ${ }^{\text {iii }}$ Again, the idea that the ACA may allow some people currently on SSI/SSDI to work by severing the tie between eligibility for cash benefits and access to health care while also keeping others from having to apply was a prominent theme.

Focusing on a single state, Dr. Jean Hall (Hall, Kurth \& Hunt, this issue) from the University of Kansas presented survey and administrative data from a sample of working-age, dually-eligible individuals in Kansas that suggested employment might result in improved health and lower health care costs over time ${ }^{\text {iv }}$. Importantly, these authors noted that employment, even at low levels, was associated with better health and health behaviors as well as lower costs. Despite these positive correlations between health and employment, study participants reported 
being discouraged from working by medical professionals and federal disability policies. These findings thus re-emphasized the potential of employment to decrease federal and state costs and improve health for duals with disabilities if policies and practices support employment efforts.

Finally, Dr. Jae Kennedy from Washington State University (Kennedy, Blogett \& Gimm, this issue) used data from the Medicare Current Beneficiary Survey (MCBS) to explore age and eligibility distribution of younger duals and the prevalence of factors associated with a return to work for this population ${ }^{\mathrm{v}}$. They found that younger Medicare beneficiaries (ages 21 to 44) have nearly 20 times greater odds of being dually eligible than other beneficiaries, but experience a lower burden of chronic illness and higher rates of high school completion than older duals. These findings reinforce the fact that many duals may be on these programs for decades and accrue very large lifetime costs, but that work is a realistic goal for many and a viable alternative to long-term program dependence.

\section{Health Outcomes Panel}

Jenna Libersky, MPH from Mathematica Policy Research (this issue) used Medicaid administrative data to document enrollment rates of duals under age 65 into different types of state Medicaid managed care programs and compared their enrollment rates and costs with Medicaid-only enrollees ${ }^{\mathrm{vi}}$. She found that enrollment for duals in any form of Medicaid managed care is one-third that of Medicaid-only beneficiaries with disabilities and that costs varied widely from state to state. Historically, states have not enrolled duals into managed care programs despite their high costs and needs, at least in part due to federal disincentives to do so. Initiatives under the ACA, however, will remove some barriers and researchers will need to stay abreast of how managed care affects outcomes for the younger dually-eligible population. 
Next, Dr. Ray Glazier (this issue) from Abt Associates presented data from the National Survey on Drug Use and Health demonstrating that people with disabilities have higher rates of cigarette smoking and abuse of prescription drugs than do their non-disabled peers ${ }^{\mathrm{vii}}$. Because both of these behaviors have a direct negative influence on the health of the population as well as their potential employability, recommendations included increasing accessibility of substance abuse treatment facilities and training for vocational rehabilitation providers on how to recognize and address these issues.

Dr. Amanda Reichard from the University of Kansas (Reichard \& Fox, this issue) presented information based on Medical Expenditure Panel Survey (MEPS) data that indicates duals with disabilities are less likely to get recommended health screenings, which may result in worse outcomes ${ }^{\text {viii }}$. In particular, they looked at people with physical disabilities, people with cognitive limitations, people with both of these conditions, and people with neither of these conditions. Overall, people with physical disabilities, alone or in combination with cognitive limitations, had the highest costs, poorest health, and lowest use of preventive services. These findings highlight the fact that duals are a heterogeneous group and that targeted interventions have the greatest likelihood of success.

Finally, Dr. Doug Bradham from the University of Kansas School of Medicine and the Robert J. Dole VA Medical Center discussed the outcomes of veterans with multiple sclerosis with a different type of dual eligibility: those dually eligible for Veterans Health Administration (VHA) services and Medicare. ${ }^{\text {ix }}$ Overall, only $8.5 \%$ of their sample from the VHA Multiple Sclerosis Surveillance Registry was dually eligible. Their preliminary findings indicate that individuals with service-related MS and those dually eligible for Medicare incur the highest costs 
to the VHA system. The authors plan to link their findings with Medicare claims data to better understand comprehensive health care utilization in this population.

\section{Summary}

More research will be needed as ACA implementation continues to assess the extent to which coverage expansions affect applications to and exits from federal disability programs. Because younger individuals dually eligible for Medicare and Medicaid are among the sickest and highest cost individuals in both programs, and because they have the potential to stay on the disability rolls for many years, their movement to employment and/or other health insurance coverage has the potential to result in great cost savings over time. Understanding personal and programmatic factors that are associated with decreased dependence on benefits and greater employment rates will be essential to crafting policies and creating targeted interventions to promote and support these changes. 
References

${ }^{i}$ Medicare Payment Advisory Committee. A Data Book: Health Care Spending and the Medicare Program, June 2012. Available from: http://www.medpac.gov/documents/Jun12DataBookEntireReport.pdf; 2012.

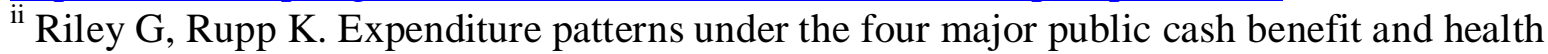
insurance programs for working-age adults with disabilities. J Disabil Pol Studies, 2012 Online First. Available from: http://dps.sagepub.com/content/early/2012/12/26/1044207312469828; 2012.

iii Levy A, Bruen B, Ku L. The potential employment impact of health reform on working-age adults with disabilities. J Disabil Pol Studies, 2012 Online First Available from http://dps.sagepub.com/content/early/2012/05/23/1011771044207312446225; 2012.

${ }^{\text {iv }}$ Hall J, Kurth N, Hunt S. Employment as a health determinant for working-age, dually-eligible people with disabilities. Disability and Health Journal XXXX

${ }^{v}$ Kennedy J, Blodgett E, Gimm G. Return to work: A critical aspect of care coordination for younger dual eligibles. Disability and Health Journal XXXX

${ }^{v i}$ Libersky J. National and state trends in enrollment and spending for dual eligibles under age 65 in Medicaid managed care. Disability and Health Journal XXXX

${ }^{\text {vii }}$ Glazier R. Recent trends in substance abuse among persons with disabilities compared to that of persons without disabilities. Disability and Health Journal XXXX

viii Reichard A, Fox M. Using Population-Based Data to Examine Preventive Services by Disability Type among Dually Eligible (Medicare/Medicaid) Adults. Disability and Health Journal XXXX

${ }^{\text {ix }}$ Bradham D, Twusami-Ankrah P, Culpepper W, Jin S, Silva A et al. Annual VHA healthcare use by dual eligible veterans with multiple sclerosis from 1998 to 2007. In prep. 